\title{
Increased risk of cerebrovascular accident related to non-alcoholic fatty liver disease: a meta-analysis
}

\author{
Jianping $\mathrm{Hu}^{1, *}$, Yong $\mathrm{Xu}^{2,{ }^{2}}$, Zemin $\mathrm{He}^{1, *}$, Hui Zhang ${ }^{1, *}$, Xiaoqing Lian ${ }^{3}$, Tiantian $\mathrm{Zhu}^{3}$, \\ Caihong Liang ${ }^{3}$ and Jun $\mathbf{L i}^{1}$ \\ ${ }^{1}$ Department of General Surgery, The Affiliated Jiangning Hospital of Nanjing Medical University, Nanjing, Jiangsu Province, P.R. China \\ ${ }^{2}$ Department of Nephrology, Huai'an Second People's Hospital and The Affiliated Huai'an Hospital of Xuzhou Medical \\ University, Huai'an, China \\ ${ }^{3}$ Department of Cardiovasology, The Affiliated Jiangning Hospital of Nanjing Medical University, Nanjing, Jiangsu Province, \\ P.R. China \\ *These authors contributed equally to this work
}

Correspondence to: Caihong Liang, email: Ich@njmu.edu.cn

Jun Li, email: lijun@njmu.edu.cn

Keywords: cerebrovascular accident; non-alcoholic fatty liver disease; meta-analysis

Received: September 14, 2017 Accepted: October 27, $2017 \quad$ Published: November 29, 2017

Copyright: Hu et al. This is an open-access article distributed under the terms of the Creative Commons Attribution License 3.0 (CC BY 3.0), which permits unrestricted use, distribution, and reproduction in any medium, provided the original author and source are credited.

\section{ABSTRACT}

Recent published studies on the association between non-alcoholic fatty liver disease (NAFLD) and cerebrovascular accident (CVA) risk have yielded conflicting findings. The aim of our study was to identify the potential association by pooling all available publications. A total of nine independent studies were included into our study. The pooled odd ratio (OR) with $95 \%$ confidence interval ( $95 \% \mathrm{CI}$ ) was calculated to weigh the strength for the relationship between NAFLD and CVA risk. We also conducted stratified analyses by study design, ethnicity and disease classification for further elucidation. The pooled results of the present meta-analysis showed that NAFLD was related to increased risk of CVA (OR $=2.32,95 \%$ CI 1.84-2.93, $P<0.001)$. Besides, NAFLD is associated with increased risk of CVA among both Caucasians (OR $=2.27,95 \%$ CI 1.77-2.90, $P<0.001)$ and Asians (OR = 2.81, 95\% CI 1.43-5.51, $P=0.003$ ). Moreover, the significant association was also observed in case-control studies (OR $=2.73,95 \%$ CI 1.67-4.48, $P<0.001)$ and cohort studies (OR $=2.22,95 \%$ CI 1.71-2.89, $P<0.001$ ), respectively. In addition, NAFLD was shown to correlate with increased risk of cerebral hemorrhage $(O R=1.85,95 \%$ CI $1.05-3.27, P=0.034)$ and the ischemic stroke ( $O R=2.51,95 \% C I 1.92-3.28, P<0.001)$. In conclusion, our findings firstly provide strong evidence for a risk effect of NAFLD on CVA development.

\section{INTRODUCTION}

Cerebrovascular accident (CVA), also known as stroke, mainly includes ischemic stroke due to lack of blood and hemorrhagic stroke due to bleeding in the brain. CVA is the second most common disease of death and the major cause of disability around the world and third to fourth leading cause of death and the main cause of disability in the United States $[1,2]$. Stroke can affect survival, mentality, emotion, or a combination of the three, and the disability caused by a stroke makes survivors decrease their employability. CVA already becomes a global severe problem and leads to heavy economic burden worldwide. A number of studies have implicated that non-alcoholic fatty liver disease (NAFLD) plays an important role in the development of CVA [3-5].

NAFLD is characterized by the presence of significant lipid accumulation in the liver parenchyma without excessive alcohol ingestion and any other liver diseases. In recent years, NAFLD has already become a familiar clinical diagnosis and its prevalence and incidence increase dramatically and consistently in the general population [6]. Due to incorrect dietary habits and sedentary lifestyle, NAFLD is frequent in populations from United States, Europe, Middle-East, Australia, China and Japan and NAFLD is a common liver condition with a prevalence 
of $20-30 \%$ globally [7]. NAFLD can be categorized into nonalcoholic steatohepatitis (NASH) and nonalcoholic fatty liver (NAFL) by histology [8]. The clinical manifestations of patients with NAFLD may range from asymptomatic, transaminase rising to cirrhosis and hepatocellular carcinoma [9]. Some studies have investigated the association between NAFLD and CVA risk. Several studies showed that NAFLD played a risk role in CVA $[10,11]$, while another study indicated that NAFLD is not independently related to ischemic stroke [5]. Thus, previously available data estimating the role of NAFLD in CVA risk are inconsistent and inconclusive, probably due to a diverse population, race, study design, and CVA classification. As a result, we for the first time carry out a meta-analysis of all currently published studies to shed some light on the contradictory findings and provide a more precise estimate for the association between NAFLD and CVA risk.

\section{RESULTS}

\section{Identification and characteristics of eligible studies}

After a comprehensive search in PubMed, Embase, Cochrane Library databases, Wanfang and CNKI databases up to Sep 30, 2017, a total of 7 publications were retrieved regarding the association between NAFLD and CVA [3-5, 10-12]. Among them, 2 publications were regarded as 4 independent studies according to different CVA [10, 11]. As a result, we included 9 individual studies. Figure 1 showed details for the inclusion of all eligible studies. Characteristics of all included studies were shown in Table 1. Among the 9 studies, 2 were in case-control design, while 7 were in cohort design. 6 independent studies were conducted among Caucasians, and 3 were among Asians.

\section{Increased risk of CVA associated with NAFLD}

Overall, the pooled OR suggested that NAFLD was significantly associated with elevated risk of CVA $(\mathrm{OR}=2.32,95 \%$ CI 1.84-2.93, $P<0.001)$ (Table 2, Figure 2). Sensitivity analysis by omitting each study did not materially modify the pooled result (data not shown).

\section{Stratified analysis by ethnicity}

We performed stratified analyses among Asians and Caucasians. Increased risk of CVA was observed related to NAFLD among both the Asian population $(\mathrm{OR}=2.81$, 95\% CI 1.43-5.51, $P=0.003)$ and the Caucasian population $(\mathrm{OR}=2.27,95 \%$ CI $1.77-2.90, P<0.001)$ (Table 2, Figure 2).

\section{Stratified analysis by study design}

The pooled result of case-control studies showed that NAFLD could increase the risk of CVA $(\mathrm{OR}=2.73$,
95\% CI 1.67-4.48, $P<0.001)$, and similar result was observed in cohort studies $(\mathrm{OR}=2.22,95 \%$ CI $1.71-2.89$, $P<0.001$ ) (Table 2, Figure 3).

\section{Stratified analysis by CVA classification}

Stratified analyses were conducted to estimate the modifying effect of NAFLD on different CVA, including ischemic stroke and cerebral hemorrhage. NAFLD was shown to be related to increased risk of cerebral hemorrhage $(\mathrm{OR}=1.85,95 \%$ CI 1.05-3.27, $P=0.034)$ and ischemic stroke $(\mathrm{OR}=2.51,95 \% \mathrm{CI} 1.92-3.28$, $P<0.001$ ), respectively (Table 2 , Figure 4).

\section{Heterogeneity analysis and publication bias risk}

As shown in Table 2, we did not find obvious between-study heterogeneity between these studies. Publication bias was not detected suggested by Begg's test $(P=0.348)$, Egger's test $(P=0.578)$ and Egger's publication bias plot(Figure 5). Visual inspection of the funnel plot suggested symmetry suggesting no publication bias (Figure 6).

\section{DISCUSSION}

To the best of our knowledge, the effect of NAFLD on CVA development has drawn close attention for the past few years. Although some studies have investigated the association between NAFLD and CVA risk, currently available data have yielded inconsistent and inconclusive findings. Two prospective cohort studies revealed that NAFLD was a good predictor of cardiovascular disease and positively related to cardiovascular disease events, including ischemic stroke and cerebral hemorrhage, suggesting that NAFLD played a risk role in CVA $[10,11]$. Reversely, Moshayedi $\mathrm{H}$ et al. demonstrated that NAFLD was not independently related to higher risk of ischemic stroke [5]. Pickhardt PJ and his colleagues clarified that liver steatosis was a biomarker for cardiovascular events, such as CVA, transient ischemic attack and myocardial infarction, but it was not an independent risk factor [4]. The inconsistent findings of previous studies might be attributed to diverse study design, ethnicity, clarification of CVA, statistical power, and types of diagnostic methods of NAFLD. Domanski JP found that no increased risk of cardiovascular disease was shown among the patients with NASH as compared with those with non-NASH fatty liver [12]. Taken together, the association of CVA with NAFLD remains not being clarified, probably owing to various populations and diverse study design. Meta-analysis could shed some light on the contradictory findings across independent studies. Therefore, we carry out this metaanalysis and perform stratified analyses by ethnicity, study design and CVA classification, respectively. The pooled results suggest that NAFLD significantly 
Table 1: Descriptive characteristics of all studies

\begin{tabular}{|c|c|c|c|c|c|c|c|c|c|c|c|c|}
\hline \multirow[b]{2}{*}{ Study } & \multirow[b]{2}{*}{ Year } & \multirow[b]{2}{*}{ Location } & \multirow[b]{2}{*}{ Ethnicity } & \multirow[b]{2}{*}{$\begin{array}{l}\text { Study } \\
\text { design }\end{array}$} & \multirow[b]{2}{*}{ Diseases } & \multicolumn{3}{|c|}{ CVA } & \multicolumn{3}{|c|}{ Control } & \multirow{2}{*}{$\begin{array}{c}\text { Quality } \\
\text { assessment } \\
\text { Score" }\end{array}$} \\
\hline & & & & & & Number & $\begin{array}{c}\text { Age } \\
\text { (year) }\end{array}$ & $\begin{array}{c}\text { Gender } \\
\text { (male/female) }\end{array}$ & Number & $\begin{array}{c}\text { Age } \\
\text { (year) }\end{array}$ & $\begin{array}{c}\text { Gender } \\
\text { (male/ } \\
\text { female) }\end{array}$ & \\
\hline Fracanzani AL & 2016 & Italy & Caucasians & Cohort & Ischemic Stroke & 6 & NR & NR & 267 & NR & NR & 7 \\
\hline Moshayedi H & 2014 & Iran & Caucasians & $\begin{array}{l}\text { Case- } \\
\text { Control }\end{array}$ & Ischemic Stroke & 110 & $66.42 \pm 11.31$ & $69 / 41$ & 110 & $66.51 \pm 11.27$ & $69 / 41$ & 7 \\
\hline El Azeem HA (1) & 2013 & Egypt & Caucasians & Cohort & Ischemic Stroke & 82 & NR & NR & 665 & NR & NR & 8 \\
\hline El Azeem HA (2) & 2013 & Egypt & Caucasians & Cohort & $\begin{array}{l}\text { Cerebral } \\
\text { Hemorrhage }\end{array}$ & 49 & NR & NR & 698 & NR & NR & 8 \\
\hline Pickhardt PJ & 2013 & Korea & Asians & Cohort & $\begin{array}{c}\text { Cerebrovascular } \\
\text { Accident }\end{array}$ & 21 & NR & NR & 1029 & NR & NR & 7 \\
\hline Domanski JP & 2012 & US & Caucasians & Cohort & Stroke & 5 & NR & NR & 372 & NR & NR & 7 \\
\hline Ying I & 2011 & canada & Caucasians & $\begin{array}{l}\text { Case- } \\
\text { Control }\end{array}$ & Ischemic Stroke & 103 & $60.4 \pm 10.8$ & $59 / 44$ & 200 & $57.9 \pm 11.0$ & $97 / 103$ & 7 \\
\hline Hamaguchi M (1) & 2007 & Japanese & Asians & Cohort & Ischemic Stroke & 12 & NR & NR & 1221 & NR & NR & 6 \\
\hline Hamaguchi M (2) & 2007 & Japanese & Asians & Cohort & $\begin{array}{c}\text { Cerebral } \\
\text { Hemorrhage }\end{array}$ & 2 & NR & NR & 1231 & NR & NR & 6 \\
\hline
\end{tabular}

NR, not reported; "Quality assessment score were assessed by the Newcastle-Ottawa Scale (NOS).

confers risk effect on CVA, and the effect of NAFLD on the susceptibility to CVA was further confirmed in the stratified analyses by race, study design and CVA classification. We provide strong evidence, for the first time, for the risk role of NAFLD in CVA.

Individuals with NAFLD, characterized by liver inflammation, are at higher risk to develop liver fibrosis and cancer [13]. Recently, the influence of inflammation in stroke has received sustaining attention, and it was proved that inflammatory mechanisms play a key role in the pathogenesis and progression of atherosclerosis and stroke [14]. Increased inflammatory cytokines have been observed in the patients with NAFLD. The activation of pro-inflammatory signaling pathways in NAFLD may lead to liver chronic inflammation [15]. A systemic inflammatory status with activation of proinflammatory

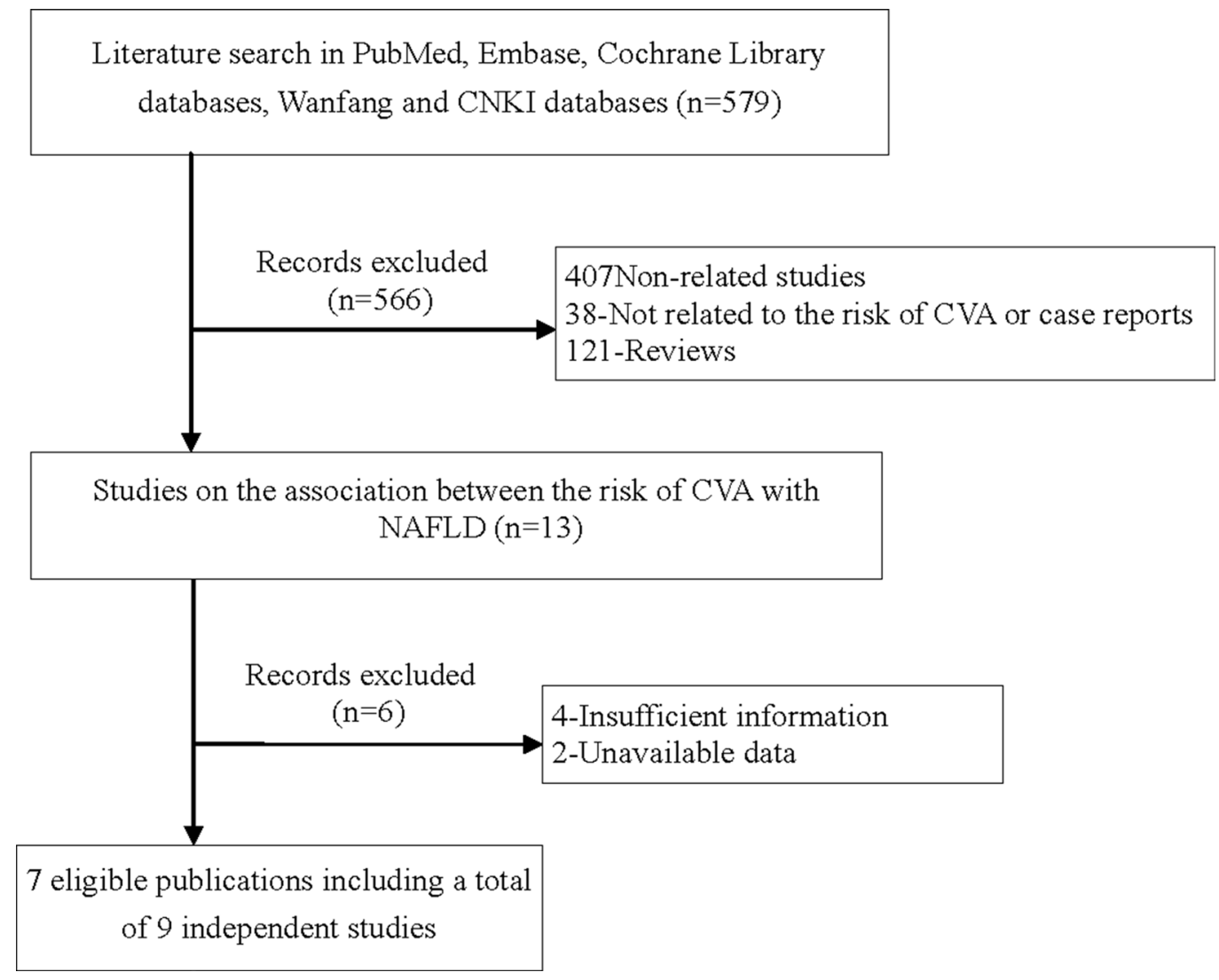

Figure 1: Flow chart for identification of eligible studies. 
Table 2: Summary meta-analysis results

\begin{tabular}{lllllc}
\hline \multirow{2}{*}{ Contrasts } & OR(95\%CI) & P value & Model & \multicolumn{2}{c}{ Heterogeneity analysis } \\
\hline Overall & $2.32(1.84-2.93)$ & $<0.001$ & $\mathrm{~F}$ & 0.0 & 0.895 \\
Caucasians & $2.27(1.77-2.90)$ & $<0.001$ & $\mathrm{~F}$ & 0.0 & 0.839 \\
Asians & $2.81(1.43-5.51)$ & 0.003 & $\mathrm{~F}$ & 0.0 & 0.569 \\
Cohort study & $2.22(1.71-2.89)$ & $<0.001$ & $\mathrm{~F}$ & 0.0 & 0.833 \\
Case-Control study & $2.73(1.67-4.48)$ & $<0.001$ & $\mathrm{~F}$ & 0.0 & 0.640 \\
Ischemic Stroke & $2.51(1.92-3.28)$ & $<0.001$ & $\mathrm{~F}$ & 0.0 & 0.828 \\
Cerebral Hemorrhage & $1.85(1.05-3.27)$ & 0.034 & $\mathrm{~F}$ & 0.0 & 0.544 \\
\hline
\end{tabular}

OR, odd ratio; $95 \% \mathrm{CI}$, 95\% confidence interval; $\mathrm{F}$, fixed-effects model.

transcription factors and infiltration of considerable proinflammatory cytokines plays a decisive role in the development of atherosclerosis and CVA $[16,17]$. The activation and upregulation of NF- $\mathrm{KB}$ resulting from hepatic steatosis will increase the expression of several proinflammatory mediators and amplify the systemic inflammation $[18,19]$. The sustained inflammatory status may promote arteriosclerosis and plaque in the arterial wall and the development of CVA. In our study, the pooled ORs suggested that NAFLD was a risk factor for CVA. However, we failed to the effect of common inflammatory mediators or transcription factors on the association between NAFLD and CVA risk due to unavailable data in previous publications.

A previous study identified that Asians and Hispanics showed a higher degree of steatosis than



Figure 2: Increased risk of cerebrovascular accident related to non-alcoholic fatty liver disease by ethnicity. OR, odd ratio; $\mathrm{CI}$, confidence interval; $\mathrm{P}$, probability. 
Whites and other ethnicities [20]. The prevalence and histological features of NAFLD also have obvious ethnic difference [21, 22]. It was well documented that the association between NAFLD and enhanced inflammation only remained significant in white people but not Chinese, African American and Hispanic people after multivariable adjustment [23]. Taken together, ethnicity may be a significant confounding factor when estimating the relationship of NAFLD with CVA risk. El Azeem HA indicates that NAFLD played a risk role in CVA [11], while Moshayedi $\mathrm{H}$ demonstrated that NAFLD is not related independently to ischemic stroke [5]. Hamaguchi $M$ proved that NAFLD was positively related to cardiovascular disease events [10], while Pickhardt PJ clarified that liver steatosis was not an independent risk factor [4]. We performed the stratified analysis by ethnicity to determine the risk of CVA related to NAFLD among different ethnic populations. The pooled results showed that increased risk of CVA was observed related to NAFLD among both Caucasian and Asian populations. Some other confounding factors may also confer modifying effects on the association between
NAFLD and CVA risk, such as study design and CVA classification. Nonetheless, stratified analysis by study design in our study showed that NAFLD could increase the risk of CVA in both case-control studies and cohort studies. However, more relevant studies are warranted for further investigation.

Ischemic stroke and cerebral hemorrhage are the main forms of CVA. We conducted the stratified analysis by CVA classification to investigate the potential role of NAFLD in different CVA mainly including ischemic stroke and cerebral hemorrhage. We found that NAFLD was related to increased risk of both ischemic stroke and cerebral hemorrhage, suggesting similar pathogenic molecular mechanisms for ischemic stroke and cerebral hemorrhage. It has been well established that hypertension is a major risk factor for developing CVA and also predisposes to the development of atherosclerosis. CVA, including cerebral infarction, cerebral hemorrhage and lacunar infarction, are the severe complications of atherosclerosis [24, 25].

Some limitations must be considered in our metaanalysis. Firstly, as mentioned above, studies regarding

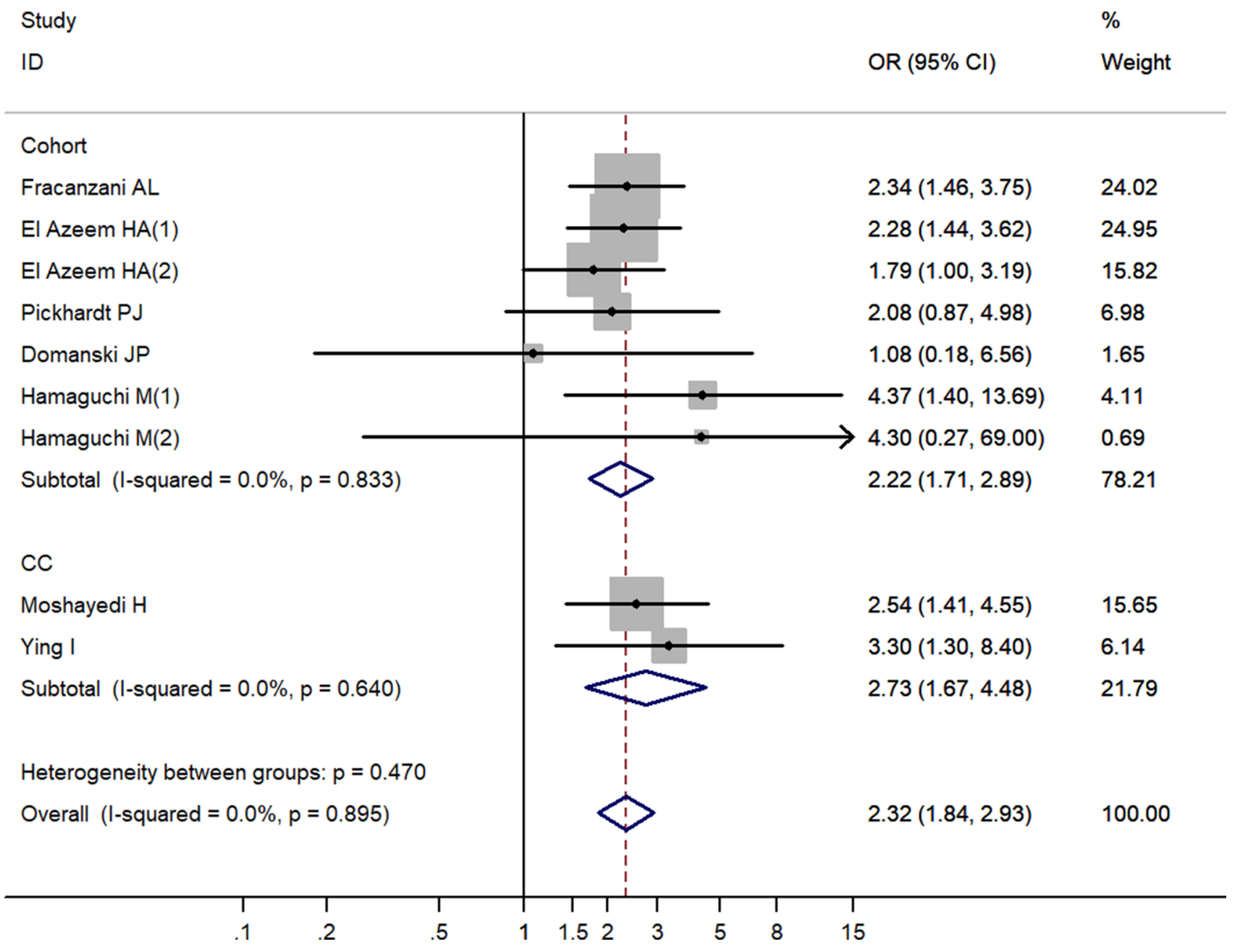

Figure 3: Increased risk of cerebrovascular accident related to non-alcoholic fatty liver disease by study design. OR, odd ratio; CI, confidence interval; $\mathrm{P}$, probability; CC, case-control study; Cohort, cohort study. 


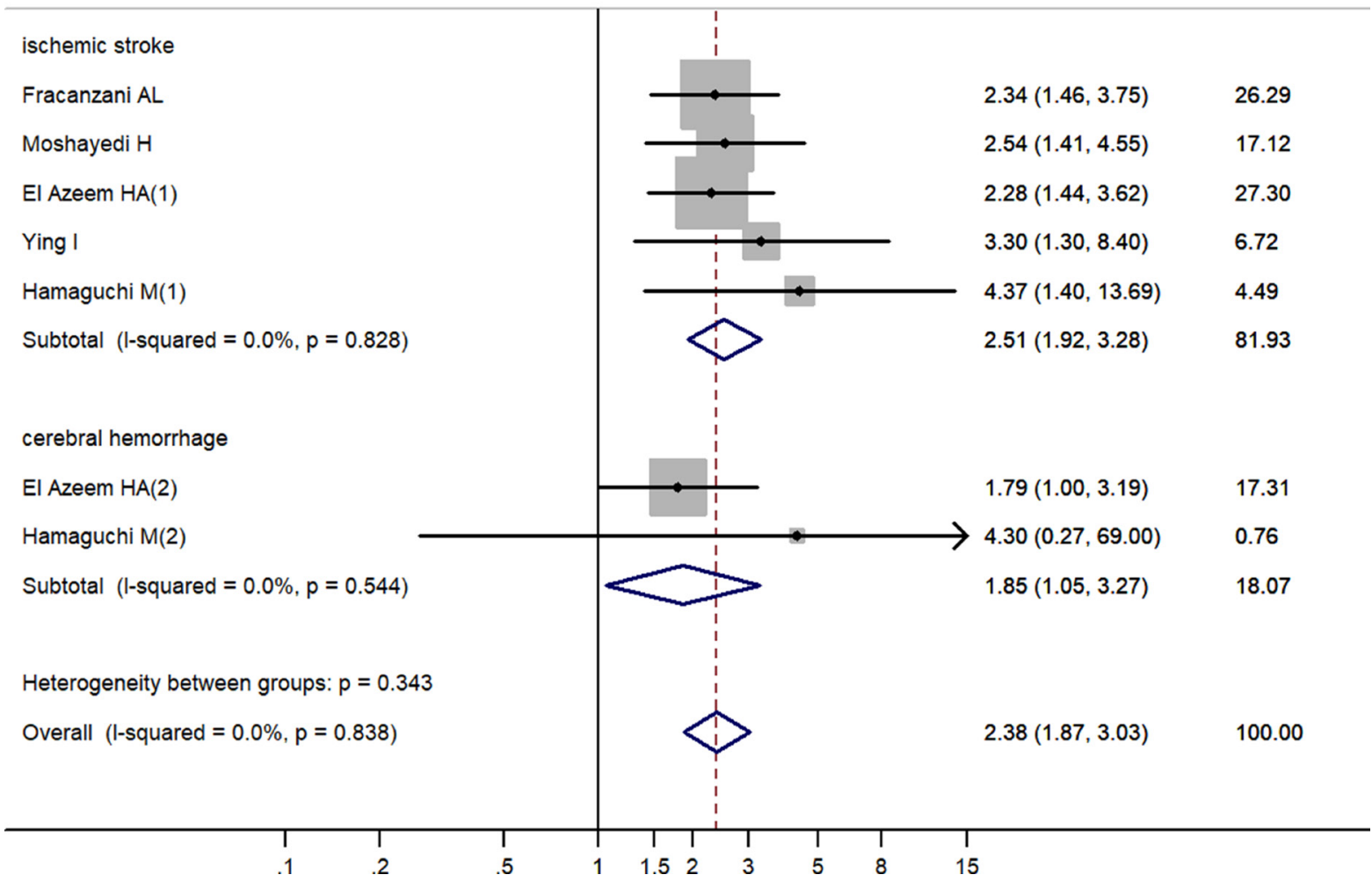

Figure 4: Increased risk of cerebrovascular accident related to non-alcoholic fatty liver disease by cerebrovascular accident classification. $\mathrm{OR}$, odd ratio; $\mathrm{CI}$, confidence interval; $\mathrm{P}$, probability.

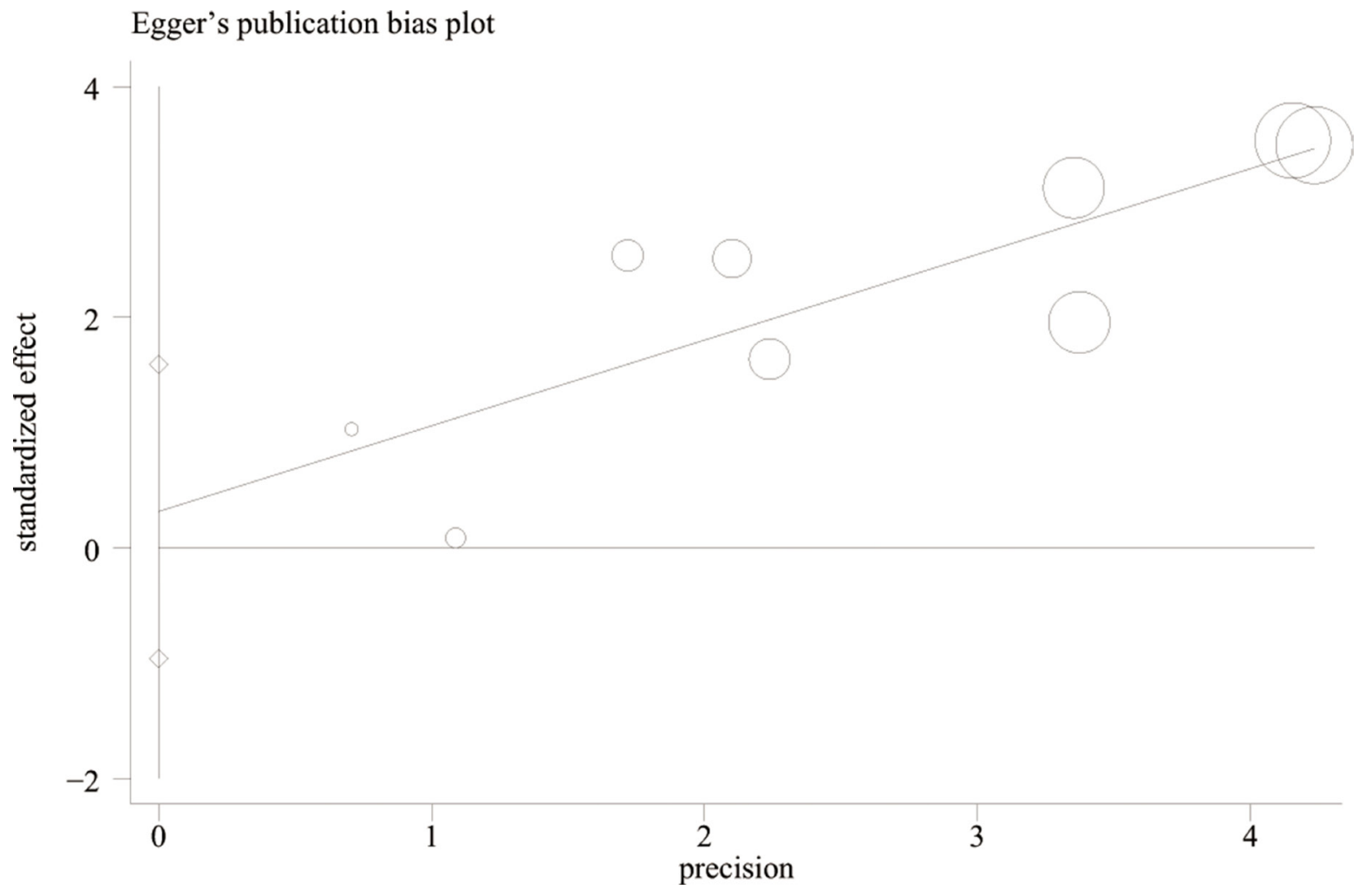

Figure 5: Egger's publication bias plot for the determination of publication bias risk $\left(P_{\text {Egger's test }}=\mathbf{0 . 5 7 8}\right)$. 
the cerebral hemorrhage risk among the Asian population were not sufficient for a precise estimate due to relatively small sample size. Secondly, the adjusted factors in every study were different, and bias might be introduced in the present study. Last but not the least, CVA is a multifactorial disease with complex pathogenic factors, including genetic polymorphisms, environmental exposures, history of the family, and so on. A combining effect between NAFLD and other common risk factors may be critical in the development of CVA, which warrants further elucidation.

Overall, the results showed that NAFLD was significantly associated with elevated risk of CVA. In the stratified analyses by ethnicity, study design and CVA classification, we also observed the similar results. Thus, our study, firstly, suggests that NAFLD is a significant risk factor for CVA among both Asians and Caucasians. However, more relevant studies are warranted for further investigation in the future.

\section{MATERIALS AND METHODS}

\section{Search strategy}

We followed the Guidelines for Meta-Analyses of Observational Studies in Epidemiology (MOOSE) group to identify eligible studies. A comprehensive literature search was performed to identify acceptable studies on the association of NAFLD and CVA risk in PubMed, Embase, Cochrane Library databases, Wanfang and CNKI databases from their inception up to Sep 30, 2017. The following terms were used: "cerebrovascular accident" or "cerebrovascular disease" or "stroke" or "ischemic stroke" or "cerebral hemorrhage" or "cerebral infarction" or "brain attack" or "paralytic"; and "fatty liver disease" or "steatohepatitis" or "hepatic steatosis". In addition, the reference lists of all retrieved studies and the PubMed link "related articles" were also screened for additional studies.

\section{Inclusion criteria}

The inclusion criteria were as follows: (1) observational studies, including case-control study and cohort study; (2) providing enough information for odds ratio (OR) or relative risk (RR) or hazard ratio (HR) and $95 \%$ confidence intervals $(95 \% \mathrm{CI})$ or raw data. Duplicate publications, reviews, case reports, irrelevant study or study with overlapping data were all excluded. The qualities of all included studies were assessed using the Newcastle-Ottawa Scale (NOS). Studies were graded as good quality if they awarded 6 to 9 stars; fair if they awarded 3 to 5 stars; and poor if they awarded less than 3 stars.

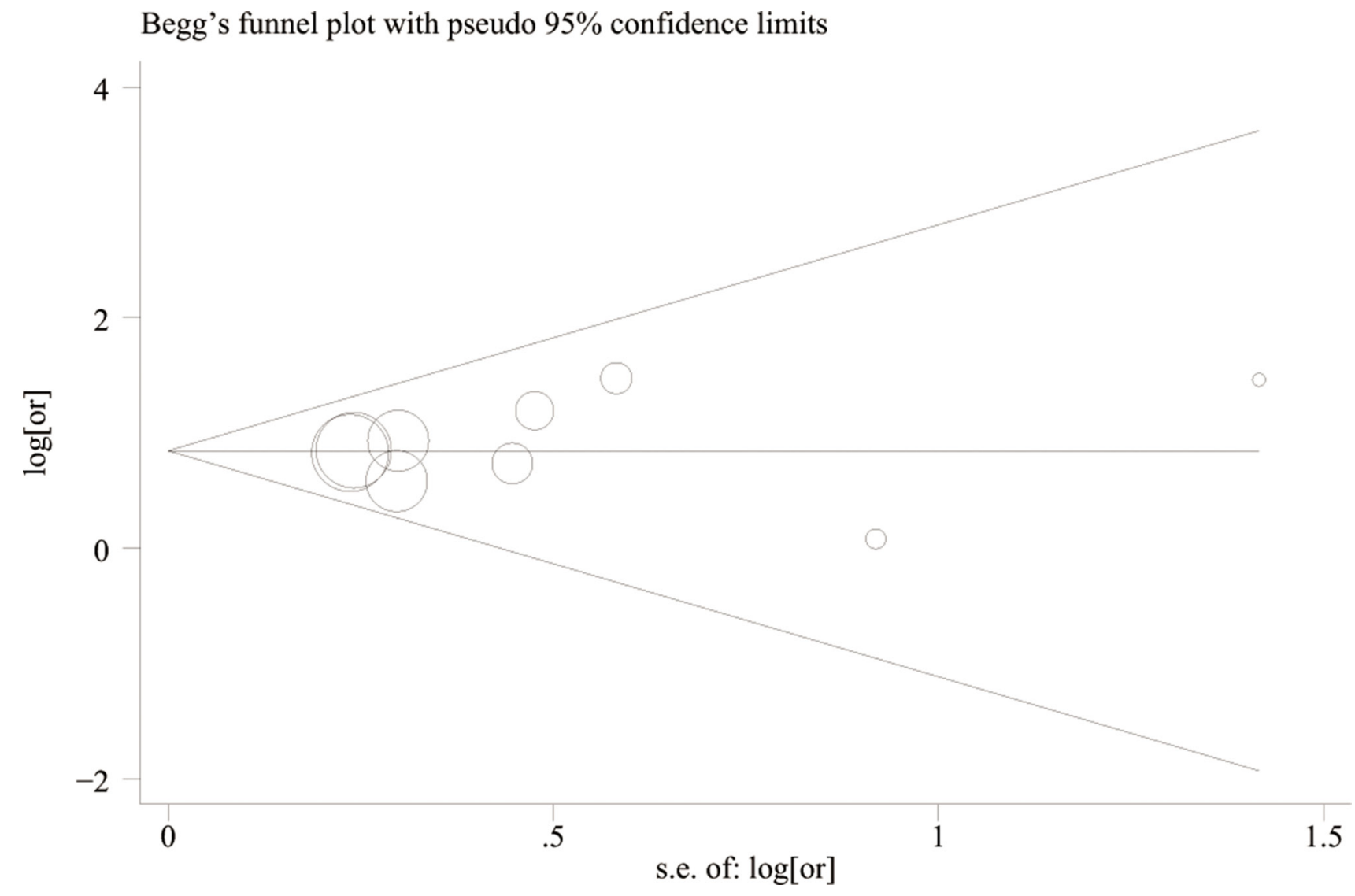

Figure 6: Begg's funnel plots for the determination of publication bias risk. P, probability; s.e, standard error; $\log [$ or], $\log [\mathrm{odd}$ ratio]. 


\section{Data extraction}

Two researchers carried out the literature search independently by using a standardized data extraction form, and the disagreements were resolved by discussion.

The data were as follows: first author, year of publication, country, ethnicity, study design, number of cases and controls, matching factors, adjusted factors, RR or HR or OR with $95 \% \mathrm{CI}$, disease classification.

\section{Statistical analysis}

The strength of the relationship between NAFLD and CVA was estimated by calculating the pooled ORs with $95 \%$ CIs. ORs and 95\% CIs were calculated with SPSS19.0 by use of the raw data in the single study. Cochrane's Q statistics and $I^{2}$ statistic tests were used to evaluate heterogeneity of the OR across studies [26, 27]. The random-effects model was applied when the betweenstudy heterogeneity was significant [28]; otherwise, the fixed-effects model was used when the between-study heterogeneity was not significant [29]. We conducted stratified analyses by study design, ethnicity and disease classification for further investigation. Sensitivity analysis was also carried out by sequential omission each study. Begg's funnel plot and Egger's test were adopted to assess the publication bias in the present meta-analysis [30, 31]. Statistical analysis was performed by using STATA 12.0 software (StataCorp, College Station, TX, USA). All $P$ values were two-sided, and $P<0.05$ suggested statistical significance.

\section{CONFLICTS OF INTEREST}

The authors declare no financial or other conflicts of interest.

\section{FUNDING}

This work was supported by Nanjing Medical Science and Technique Development Foundation (YKK16235), Nanjing Medical University Science and Technology Development Foundation (2016NJMUZD089) and Jiangning Science and Technology Project(2016Dc08).

\section{REFERENCES}

1. Donnan GA, Fisher M, Macleod M, Davis SM. Stroke. Lancet. 2008; 371:1612-1623.

2. Towfighi A, Saver JL. Stroke declines from third to fourth leading cause of death in the United States: historical perspective and challenges ahead. Stroke. 2011; 42:2351-2355.

3. Ying I, Saposnik G, Vermeulen MJ, Leung A, Ray JG. Nonalcoholic fatty liver disease and acute ischemic stroke. Epidemiology. 2011; 22:129-130.
4. Pickhardt PJ, Hahn L, Munoz del Rio A, Park SH, Reeder SB, Said A. Natural history of hepatic steatosis: observed outcomes for subsequent liver and cardiovascular complications. AJR Am J Roentgenol. 2014; 202:752-758.

5. Moshayedi H, Ahrabi R, Mardani A, Sadigetegad S, Farhudi M. Association between non-alcoholic fatty liver disease and ischemic stroke. Iran J Neurol. 2014; 13:144-148.

6. Bellentani S, Scaglioni F, Marino M, Bedogni G. Epidemiology of non-alcoholic fatty liver disease. Dig Dis. 2010; 28:155-161.

7. Masarone M, Federico A, Abenavoli L, Loguercio C, Persico M. Non alcoholic fatty liver: epidemiology and natural history. Rev Recent Clin Trials. 2014; 9:126-133.

8. Chalasani N, Younossi Z, Lavine JE, Diehl AM, Brunt EM, Cusi K, Charlton M, Sanyal AJ; American Gastroenterological Association; American Association for the Study of Liver Diseases; American College of Gastroenterologyh. The diagnosis and management of non-alcoholic fatty liver disease: practice guideline by the American Gastroenterological Association, American Association for the Study of Liver Diseases, and American College of Gastroenterology. Gastroenterology. 2012; 142:1592-1609.

9. Mulhall BP, Ong JP, Younossi ZM. Non-alcoholic fatty liver disease: an overview. J Gastroenterol Hepatol. 2002; 17:1136-1143.

10. Hamaguchi M, Kojima T, Takeda N, Nagata C, Takeda J, Sarui H, Kawahito Y, Yoshida N, Suetsugu A, Kato T, Okuda J, Ida K, Yoshikawa T. Nonalcoholic fatty liver disease is a novel predictor of cardiovascular disease. World J Gastroenterol. 2007; 13:1579-1584.

11. El Azeem HA, Khalek el SA, El-Akabawy H, Naeim H, Khalik HA, Alfifi AA. Association between nonalcoholic fatty liver disease and the incidence of cardiovascular and renal events. J Saudi Heart Assoc. 2013; 25:239-246.

12. Domanski JP, Park SJ, Harrison SA. Cardiovascular disease and nonalcoholic fatty liver disease: does histologic severity matter? J Clin Gastroenterol. 2012; 46:427-430.

13. Dietrich P, Hellerbrand C. Non-alcoholic fatty liver disease, obesity and the metabolic syndrome. Best Pract Res Clin Gastroenterol. 2014; 28:637-653.

14. McColl BW, Allan SM, Rothwell NJ. Systemic infection, inflammation and acute ischemic stroke. Neuroscience. 2009; 158:1049-1061.

15. Tarantino G, Caputi A. JNKs, insulin resistance and inflammation: A possible link between NAFLD and coronary artery disease. World J Gastroenterol. 2011; 17:3785-3794.

16. Dowman JK, Tomlinson JW, Newsome PN. Pathogenesis of non-alcoholic fatty liver disease. QJM. 2010; 103:71-83.

17. Perazzo H, Poynard T, Dufour JF. The interactions of nonalcoholic fatty liver disease and cardiovascular diseases. Clin Liver Dis. 2014; 18:233-248.

18. Stefan N, Kantartzis K, Haring HU. Causes and metabolic consequences of Fatty liver. Endocr Rev. 2008; 29:939-960. 
19. Cai D, Yuan M, Frantz DF, Melendez PA, Hansen L, Lee J, Shoelson SE. Local and systemic insulin resistance resulting from hepatic activation of IKK-beta and NF-kappaB. Nat Med. 2005; 11:183-190.

20. Mohanty SR, Troy TN, Huo D, O’Brien BL, Jensen DM, Hart J. Influence of ethnicity on histological differences in nonalcoholic fatty liver disease. J Hepatol. 2009; 50:797-804.

21. Browning JD, Szczepaniak LS, Dobbins R, Nuremberg P, Horton JD, Cohen JC, Grundy SM, Hobbs HH. Prevalence of hepatic steatosis in an urban population in the United States: impact of ethnicity. Hepatology. 2004; 40:13871395.

22. Weiskirchen R, Wasmuth HE. The genes that underlie fatty liver disease: the harvest has begun. Hepatology. 2009; 49:692-694.

23. Al Rifai M, Silverman MG, Nasir K, Budoff MJ, Blankstein R, Szklo M, Katz R, Blumenthal RS, Blaha MJ. The association of nonalcoholic fatty liver disease, obesity, and metabolic syndrome, with systemic inflammation and subclinical atherosclerosis: The Multi-Ethnic Study of Atherosclerosis (MESA). Atherosclerosis. 2015; 239:629633.
24. Sierra C, Coca A, Schiffrin EL. Vascular mechanisms in the pathogenesis of stroke. Curr Hypertens Rep. 2011; 13:200-207.

25. Sierra C. Essential hypertension, cerebral white matter pathology and ischemic stroke. Curr Med Chem. 2014; 21:2156-2164.

26. Cochran WG. The comparison of percentages in matched samples. Biometrika. 1950; 37:256-266.

27. Higgins JP, Thompson SG, Deeks JJ, Altman DG. Measuring inconsistency in meta-analyses. BMJ. 2003; 327:557-560.

28. DerSimonian R, Laird N. Meta-analysis in clinical trials. Control Clin Trials. 1986; 7:177-188.

29. Mantel N, Haenszel W. Statistical aspects of the analysis of data from retrospective studies of disease. J Natl Cancer Inst. 1959; 22:719-748.

30. Egger M, Davey Smith G, Schneider M, Minder C. Bias in meta-analysis detected by a simple, graphical test. BMJ. 1997; 315:629-634.

31. Stuck AE, Rubenstein LZ, Wieland D. Bias in meta-analysis detected by a simple, graphical test. Asymmetry detected in funnel plot was probably due to true heterogeneity. BMJ. 1998; 316:469; author reply 470-461. 